\title{
Office hysteroscopy in women with postmenopausal bleeding: see and treat of endometrial polyps using a Duckbill Polyp Snare
}

Published online: 11 May 2004

(C) Springer-Verlag Berlin / Heidelberg 2004

\begin{abstract}
In women with postmenopausal bleeding, intracavitary pathology is often diagnosed. Hysteroscopy can be used as a diagnostic tool for intracavitary abnormalities. Nowadays, office hysteroscopy can be used as a therapeutic tool as well. We describe the use of office hysteroscopy in the diagnosis and treatment in women with postmenopausal bleeding. In 83 patients with postmenopausal bleeding in whom an endometrium thickness of more than $4 \mathrm{~mm}$ was found, hysteroscopy was performed. Hysteroscopy was performed in an office setting, using a vaginoscopic approach. If a polyp was visualised, this polyp was removed in the same session (see and treat) using the Duckbill Polyp Snare. In 10.8\% of the patients, endometrial carcinoma was diagnosed. In $41 \%$ of the patients, the polyp was diagnosed and treated. Office hysteroscopy in women with postmenopausal bleeding and an endometrium thickness of more than $4 \mathrm{~mm}$ offers the possibility of diagnosis as well as treatment in the same session by using a polyp snare.
\end{abstract}

Keywords Office hysteroscopy · Duckbill Polyp Snare . Postmenopausal bleeding

\section{Introduction}

Between 10 and $15 \%$ of women with postmenopausal bleeding have endometrial carcinoma or a premalignant disorder of the endometrium $[1,2]$. Transvaginal ultrasound with measurement of endometrial thickness can be used to discriminate between a normal and pathological endometrium. When a cut-off level of $5 \mathrm{~mm}$ is used, a prevalence of endometrial polyps of up to $43 \%$ can be found [3]. Women with postmenopausal bleeding undergo endometrial biopsy to exclude endometrial cancer. Women who are biopsied and are found to have either "benign diagnosis" or "insufficient tissue for diagnosis" may still

A. Timmermans $(\square) \cdot$ S. Veersema

St. Antonius Hospital,

Nieuwegein, The Netherlands

e-mail: anne_timmermans@hotmail.com have endometrial polyps. Endometrial cancer is then excluded, but benign polyps are not diagnosed or treated.

Hysteroscopy with endometrial resection has been proven to be superior to dilatation and curettage, especially in the presence of focal lesions [3]. Nowadays, the diagnostic and therapeutic utility of hysteroscopy in intracavitary pathologies is widely recognised. Improvements in endoscopic technology and the introduction of office hysteroscopy have made minimally invasive options available for both the diagnosis and treatment of endometrial polyps [4]. We describe the use of office hysteroscopy in the diagnosis and treatment of women with postmenopausal bleeding.

\section{Subjects and methods}

\section{Patients}

From January 2002 until December 2002, women with postmenopausal bleeding presenting at our clinic underwent transvaginal ultrasound examination. When an endometrium thickness of more than $4 \mathrm{~mm}$ was found on ultrasound, these women were scheduled for office hysteroscopy. In 2002, 83 women underwent office hysteroscopy because of postmenopausal bleeding and an endometrium thickness of more than $4 \mathrm{~mm}$.

Office hysteroscopy

Office hysteroscopy was performed using a 5.5-mm rigid continuous-flow hysteroscopic system with a 5-Fr. working channel (Olympus). A vaginoscopic approach to hysteroscopy was used, i.e., without a speculum, tenaculum, dilatation or anaesthesia [5]. The uterine cavity was distended using sorbitol. If during hysteroscopy a lesion suspect for malignancy was seen, this lesion was biospsied. If no lesion was seen, a random biopsy from the endometrium was taken. If a focally growing (benign) lesion was identified (a polyp), if possible this lesion was removed in the same session (see and treat). Small ( $<5 \mathrm{~mm}$ ) polyps were removed using mechanical instruments such as endoscopic forceps or scissors; polyps larger than $5 \mathrm{~mm}$ were removed using the Duckbill Polyp Snare. 


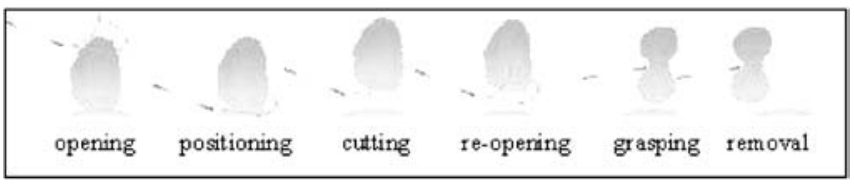

Fig. 1 The Duckbill Polyp Snare

Operative technique: the Duckbill Polyp Snare

The snare ( 5 Fr.) was introduced through the working channel of the scope until the tip was visualised and advanced to the area of the polyp (Fig. 1). The loop was opened to hang the polyp. By moving the tip of the sheath and advancing the snare, the loop was placed to the base of the polyp. Then after closing the loop tightly, the wire was charged with an appropriate cutting current $(70 \mathrm{~W})$, thereby cutting the polyp at its base. The loop was then opened again to catch the floating polyp, and after closing the loop firmly around the free polyp, the scope was removed with the polyp, simultaneously ending the procedure. In case of larger polyps in which the base of the polyp could not be visualised or reached, the procedure was repeated until the polyp was removed completely. All tissue was sent for histological examination. The final diagnosis for each woman was made on the basis of the histological diagnosis. In case of (pre)malignancy, the patient was scheduled for further treatment.

\section{Results}

In 2002,83 patients underwent office hysteroscopy because of postmenopausal bleeding and an endometrium thickness of more than $4 \mathrm{~mm}$ on ultrasound. The mean age of the women was 60 years (range 46-88). Of these 83 women, $10.8 \%$ of the patients had an endometrial carcinoma, whereas $41 \%$ had an endometrial polyp (Table 1).

Table 1 Hysteroscopy results

\begin{tabular}{lcc}
\hline & No. & $\%$ \\
\hline Atrophy & 12 & 14.4 \\
Active benign & 12 & 14.4 \\
Endometrial polyp & 34 & 41 \\
Endocervical polyp & 4 & 4.8 \\
Submucous myoma & 6 & 7.2 \\
Hyperplasia & 4 & 4.8 \\
Atypical hyperplasia & 1 & 1.2 \\
Endometrial carcinoma & 9 & 10.8 \\
Cervical carcinoma & 1 & 1.2 \\
Total & 83 & 100 \\
\hline
\end{tabular}

In 35 cases, a polyp was identified. Of the 35 polyps, 20 polyps were removed with the Duckbill Polyp Snare. This accounted for $60 \%$ of the polypectomies; nine polyps were removed with the use of mechanical instruments, and five polyps were biopsied. Of these 35 polyps, in 34 cases a benign endometrial polyp was confirmed by histology. In one case, however, a carcinoma was found inside the polyp. This accounts for $2.8 \%$ of malignancy inside polyps.

In $46 \%$ of the women, a polyp (endocervical or endometrial) was identified and treated in the same session. In $13 \%$ of the women, a (pre)malignant lesions was seen, and hysteroscopy served for diagnosis and tissue sampling. After hysteroscopy and histology results, the patients could be scheduled for further treatment. In $41 \%$ of the women, intracavitary pathology could be excluded by hysteroscopy.

\section{Conclusion}

Office hysteroscopy in women with postmenopausal bleeding and an endometrium thickness of more than $4 \mathrm{~mm}$ offers the possibility of diagnosis as well as treatment in the same session. A decision analysis regarding the diagnosis and treatment in women with postmenopausal bleeding is necessary.

\section{References}

1. Emanuel MH, Verdel MJ, Wamsteker K, Lammes FB (1995) An audit of true prevalence of intrauterine pathology: the hysteroscopic findings, controlled for patient selection in 1,202 patients with abnormal uterine bleeding. Gynaecol Endosc 4:237-241

2. Dijkhuizen FPHLJ, Brölmann Ham, Potters A, et al (1996) The accuracy of transvaginal ultrasonography in the diagnosis of endometrial abnormalities. Obstet Gynecol 87:345-349

3. Epstein E, Ramirez A, Skoog L, Valentin L (2002) Dilatation and curettage fails to detect most focal lesions in the uterine cavity in women with postmenopausal bleeding. Acta Obstet Gynecol (Scand) 80:1131-1136

4. Bettocchi S, Ceci O, Di Venere R, et al (2002) Advanced operative office hysteroscopy without anesthesia: analysis of 501 cases treated with a 5 Fr. bipolar electrode. Hum Reprod $17: 2435-2438$

5. Bettocchi S, Selvaggi L (1997) A vaginoscopic approach to reduce the pain of office hysteroscopy. J Am Assoc Gynecol Laparosc 4:255-258 